ziehung zwischen RNS-Umsatz und der Intensität der Proteinsynthese besteht ${ }^{9}$. Nach Sтгсн und Н̈̈мMERLING ${ }^{10}$ findet eine lebhafte RNS-Synthese im Nukleolus nur in physiologisch aktiven Kernen statt. Dies erklärt dann auch die bei unseren Versuchen beobachtete allmähliche Rückbildung der Nukleoli in den Zellen, die HR oder DK ausgesetzt sind. In den betreffenden Zellen ist die Proteinsynthese reduziert. Betont sei nochmals, daß im Gegensatz zu den Kernen die voll ausgebildeten Zellen bei allen Umsetzversuchen ihre Größe und Form weitgehend bei-

9 J. Davidsox, The Biochemistry of Nucleic Acids; Methnen London [1951]. behalten. Es kommt also zu einer reversiblen Änderung der „Kern-Plasma-Relation“.

Die vom BL abhängige photochemische Reaktion, deren Auswirkung wir hier beobachteten, läuft offenbar in den Randbezirken des Plasmas ab ${ }^{4}$. Von dort aus kann also das Kernverhalten kontrolliert werden. Die vorliegende Arbeit zeigt demnach, daß durch photochemische Reaktionen, die im Plasma ablaufen, die Aktivität des Kerns entscheidend gesteuert werden kann.

10 H. Stich u. J. Hämmerling, Z. Naturforschg. 8 b, 329 [1953].

\title{
Elektronenmikroskopische Beobachtungen an der generativen Zelle von Oenothera hookeri Torr. et Gray
}

\author{
Von Lothar Diers \\ Aus dem Botanischen Institut der Universität zu Köln \\ (Z. Naturforschg. 18 b, 562-566 [1963] ; eingegangen am 20. Dezember 1962)
}

\begin{abstract}
In the germinating pollen grain the generative cell shows the structure of a meristematic cell. It is separated from the cytoplasm of the vegetative cell by a clearly visible wall, on the average about $30-60 \mathrm{~m} \mu$ thick. This wall appears to be formed by two darkly stained membranes including a lighter region of varying thickness. In the generative cell there are a big nucleus, plastids which show a scarcely developed lamellar system but no starch grains, mitochondria, dictyosomes, very probably lipid bodies and unidentified cytoplasmic inclusion bodies. The endoplasmic reticulum is continuous with the membranes of the nuclear encelope and extends through the cytoplasmic substance in which no vacuoles are recognizable.
\end{abstract}

Seit den Arbeiten von Strasburger ${ }^{1}$ sind mehrfach Versuche unternommen worden, die Struktur der generativen Zelle bei den Angiospermen mit Hilfe des Lichtmikroskops und bestimmter Fixierungs- und Färbungsmethoden aufzuklären ${ }^{2}$. Zunächst stand die Frage nach dem Bau der generativen Zelle nur unter cytologischem Aspekt. Sie erhielt in genetischer Hinsicht Bedeutung, als Renner ${ }^{3}$ bei der Erklärung der von ihm gefundenen Bastardscheckung, die nach Kreuzung bestimmter OenotheraArten auftritt, zu der Folgerung kam, daß bei der Befruchtung nicht nur der Spermakern, sondern auch Plasmateile, in diesem Fall Plastiden, in die Eizelle gelangen müssen. Da eine solche Übertragung möglicherweise durch Spermazellen erfolgen kann, suchte man in diesen und in der generativen Zelle, aus der sie unmittelbar hervorgehen, nach

1 E. Strasburger, Jenaische Z. Naturwiss. 11, 435 [1877].

2 Zusammenfassende Darstellung u. a. bei K. Schnarf, Vergleichende Cytologie des Geschlechtsapparates der Kormo-
Plastiden und anderen Organellen. Hinsichtlich der Struktur der generativen Zelle stellten sich so mehrere Fragen, von denen nur zwei herausgegriffen seien: 1. Liegt im Pollenkorn und Pollenschlauch immer eine generative Zelle oder nur ein generativer Kern? 2. Wenn eine wirkliche Zelle vorhanden ist, wie ist diese dann gegen das vegetative Plasma abgegrenzt, und welche Organellen kommen in ihr vor? Die lichtmikroskopischen Untersuchungen vermochten nicht alle diese Fragen befriedigend zu beantworten. Das Vorhandensein von Plasmabestandteilen, wie Plastiden und Mitochondrien, in der generativen Zelle konnte bis heute überhaupt nicht oder in fast allen Fällen nur unsicher nachgewiesen werden. Die Schwierigkeit, eindeutige Beobachtungen vorzunehmen, läßt sich vor allem durch die Tatsache erklären, daß die Größe der fraglichen Par-

phyten, Berlin 1941; P. Maheshwari, Bot. Rev. 15, l [1949].

3 O. Rexier, Z. Vererbungsl. 27, 235 [1922]. 
tikel, die häufig an der Grenze des lichtmikroskopischen Auflösungsvermögens liegt, eine sichere Unterscheidung aus methodischen Gründen nicht ermöglicht. Daher erscheint eine elektronenmikroskopische Untersuchung angebracht, um Klarheit über den Bau der generativen Zelle zu gewinnen.

Arbeiten dieser Art wurden bisher nur an Monokotylen durchgeführt. So unternahm Chardard ${ }^{4}$ Studien an Pollenkörnern einiger Orchideen und Bopp-HASSENкамР $^{5}$ an Pollenschläuchen von Lilium candidum und Fritillaria imperialis. Der zuerst genannte Autor fand in der generativen Zelle neben dem großen Kern nur wenige plasmatische Bestandteile: Golgi-Körper, Strukturen des endoplasmatischen Retikulums und Vakuolen. Mitochondrien und Plastiden kommen nicht vor. Dieses Ergebnis wird in einer kürzlich veröffentlichten Arbeit bestätigt ${ }^{6}$. Bopp-Hassenkamp ${ }^{5}$ stellte im Plasma der generativen Zelle oder der Spermazellen, die sie nicht zu unterscheiden vermochte, das Vorkommen von Plastiden, Mitochondrien und Teilen des endoplasmatischen Retikulums fest. Wohl in Folge der angewandten Fixierung mit $\mathrm{OsO}_{4}$ und nachfolgender Einbettung in Methacrylat sind auf den Abbildungen die beschriebenen Zellorganellen sowie die Wandung der generativen Zelle nicht immer deutlich zu erkennen.

Die vorliegende Untersuchung sollte an einer Oenothera-Art erfolgen, da nach den genetischen Befunden von Renner ${ }^{7}$, Schwemmle und Mitarbb. ${ }^{8}$, Sснӧтz ${ }^{9}$, Stubbe ${ }^{10}$ in dieser Gattung eine Übertragung von Plasmateilen aus dem männlichen Gametophyten in die Eizelle anzunehmen ist. So erschien es gerade in diesem Genus wünschenswert zu erfahren, welche Organellen in der generativen Zelle vorkommen. Als Species wurde Oenothera hookeri gewählt, da Menke ${ }^{11}$ bereits bei dieser Art elektronenmikroskopische Beobachtungen über die Plastidenentwicklung im Sproß- und Wurzelmeristem vorgenommen hatte. Außerdem führte schon früher Kaienburg ${ }^{12}$ eingehende lichtmikroskopische Studien an Pollenkörnern und Pollenschläuchen verschiedener Oenothera-Species durch, so daß ein Vergleich ihrer Ergebnisse mit den hier beschriebenen möglich ist.

\section{Material und Methode}

Auf die Spitzen von Narbenästen, die reichlich Sekret abgesondert hatten, wurde Pollen aufgetragen. Die Pollenkörner keimten nach kurzer Zeit und hatten

4 R. Chardard, Revue Cytol. 19, 233 [1958].

5 G. Bopp-Hassenkamp, Z. Naturforschg. 15 b, 91 [1960].

6 R. Chardard, Revue Cytol. 24, 1 [1962].

7 O. Renner, Flora [Jena] 130, 218 [1936].

8 J. Schwemmle u. Mitarb., Z. Vererbungsl. 75, 358 [1938].

9 F. Sснӧтz, Planta 43, 182 [1954].

10 W. Stubbe, Z. Bot. 48, 191 [1960]. nach 20 Min. Schläuche ausgetrieben, die zum Teil schon in das Narbengewebe eindrangen. Nach etwa 25 Min. wurde der vorderste Teil der Narbenspitze abgetrennt und in $\mathrm{KMnO}_{4}$-Lösung fixiert und kontrastiert. Da vor allem die Membranstrukturen der Zelle erhalten und hervorgehoben werden sollten, schien $\mathrm{KMnO}_{4}$, wie zahlreiche Arbeiten seit den Befunden von LufT ${ }^{13}$ gezeigt haben, als Fixierungs- und Kontrastierungsmittel am besten geeignet. Im einzelnen kam 2-proz. und 4-proz. $\mathrm{KMnO}_{4}$-Lösung mit zweistündiger sowie 2-proz. $\mathrm{KMnO}_{4}$-Lösung mit vierstündiger Einwirkungsdauer zur Anwendung. Fixierung, Entwässerung und Einbettung in Methacrylat erfolgte im wesentlichen nach der von Menke ${ }^{11,14}$ übernommenen Arbeitsweise. Auf einem Leitz-Ultramikrotom nach Fernández-Morán wurde mit Glas- und Diamantmessern geschnitten. Ein Siemens Elmiskop I diente zur Anfertigung der Aufnahmen.

\section{Bau der generativen Zelle}

Alle hier wiedergegebenen Aufnahmen zeigen Ausschnitte von auskeimenden Pollenkörnern. Der vegetative Kern und die generative Zelle, die in den meisten Fällen langgestreckt oder spindelförmig gestaltet ist, liegen noch innerhalb des Pollenkorns. Die erst im Pollenschlauch erfolgende Teilung der generativen Zelle in die beiden Spermazellen hat noch nicht stattgefunden.

In der Mitte der Übersichtsaufnahme (Abb. ${ }^{*}$ ) hebt sich von dem mit zahlreichen Vakuolen durchsetzten Plasma ein großes, zerlapptes Gebilde ab. Es stellt den vegetativen Kern dar. In der oberen rechten Ecke des Bildes liegt die generative Zelle. Sie ist annähernd quer durchschnitten. Eine Wandung grenzt sie gegen das vegetative Plasma ab. Der große Kern füllt auf diesem Schnitt die Zelle fast ganz aus. Das generative Plasma zeigt einige Organellen, die bei der geringen Vergrößerung nicht eindeutig bestimmbar sind. Die vakuolenfreie cytoplasmatische Grundsubstanz erscheint homogen und dicht. Im vegetativen Plasma erkennt man Leukoplasten mit dunkel kontrastierten Stärkekörnern sowie schwarze, manchmal ringförmige Gebilde ( $x$ ), die sehr wahrscheinlich Lipoidtropfen darstellen. Weitere Bestandteile, wie Mitochondrien. GolgiKörper, Strukturen des endoplasmatischen Retiku-

11 W. Menke, Z. Naturforschg. 14 b, 394 [1959]; Z. Naturforschg. 15 b, 479 [1960].

12 A. Kaienburg, Planta 38, 377 [1950].

13 J. H. Luft, J. biophysic. biochem. Cytol. 2, 799 [1956].

14 W. MenKe, Z. Naturforschg. 12 b, 654 [1957].

* Abb. 1-8 s. Tafel S. 564 a, b, c und d. 
lums sind bei dieser schwachen Vergrößerung nicht deutlich sichtbar. Die vegetative Zelle zeigt sich dicht vollgepackt mit Organellen. Selten erkennt man größere Bezirke, so in unmittelbarer Nähe der generativen Zelle, in denen sich nur homogen erscheinende cytoplasmatische Grundsubstanz befindet. Auf die einzelnen Bestandteile in der vegetativen Zelle sei hier nicht weiter eingegangen, da eine ausführliche Analyse in einer noch nicht abgeschlossenen Untersuchung folgt.

Zellwand: Die generative Zelle hebt sich deutlich durch ihre Wandung und ihren großen Kern von dem vakuolisierten vegetativen Plasma ab. Die verhältnismäßig dünne Wand, deren Dicke durchschnittlich nur $30-60 \mathrm{~m} \mu$ mißt, umschließt den Kern sowie das generative Plasma vollständig (Abb. 2) . Ihr Verlauf ist z. T. unregelmäßig; Fortsätze können in das vegetative Plasma hineinragen (Abb. 4). Die Wandung zeigt an den Stellen, wo sie schräg geschnitten wurde, eine verwaschene Kontur; dagegen dort, wo sie senkrecht durchschnitten wurde, erkennt man ihren Aufbau. Zwei stark kontrastierte Linien umgeben einen mittleren Streifen, der besonders nach Fixierung mit 4-proz. $\mathrm{KMnO}_{4}$-Lösung dunkler erscheint als die cytoplasmatische Grundsubstanz. Gelegentlich treten runde oder ovale Einschlüsse in der Wandung auf. Wie Serienschnitte beweisen, handelt es sich um röhrenförmige Einstülpungen des generativen Plasmas (Abb.6). Eine Verbindung dieser Einschlüsse auch mit dem vegetativen Plasma konnte nicht beobachtet werden. Ein dunkler Streifen, der eine sehr veränderliche Breite aufweist und sich verzweigt, kann die Wandung von der Seite des vegetativen Plasmas her umgeben. So entsteht manchmal der Eindruck, als ob eine zweite, die generative Zelle umschließende Hülle vorliegt. Diese Strukturen vereinigen sich nicht mit der Wand der generativen Zelle. Sie stellen keine dünnen Platten oder Stränge dar, sondern besitzen eine oft beträchtliche Tiefe, d.h. eine Ausdehnung senkrecht zur Bildebene (Abb. 6), und gehören dem endoplasmatischen Retikulum der vegetativen Zelle an. - Der Arbeit von Bopp-HassenKAмP ${ }^{5}$ kann man entnehmen, daß die Wandung der generativen Zelle als eine Art von Plasmalemma anzusehen sei. Entgegen dieser Auffassung halten wir bei dem hier untersuchten Objekt die Wandung

15 W. Menke, Portugal. Acta Biol., Sér. A 6, 243 [1962].

16 W. G. Whaley, H. H. Mollenhauer u. J. H. Leech, Amer. J. Bot. 47, 401 [1960]. wegen ihrer Dicke und stärkeren Kontrastierbarkeit für eine Zellwand, der sowohl vom generativen wie vom vegetativen Plasma her je ein Plasmalemma anliegt. Die Wandung der generativen Zelle besitzt ein ähnliches Aussehen wie die „doppelte Cytoplasmamembran", die Menke ${ }^{15}$ bei der Autosporenbildung von Prototheca ciferrii beschrieb.

Kern und endoplasmatisches Retikulum: Der Kern füllt einen großen Teil der generativen Zelle aus. In seinem Innern lassen sich nach der angewandten Fixierung keine Strukturen erkennen. Die Kernmembran weist außer Poren an einigen Stellen Unterbrechungen auf, wie sie in ähnlicher Ausbildung Whaley et al. ${ }^{16}$ als ,discontinuities in the nuclear envelope" beschrieben. Das endoplasmatische Retikulum steht in unmittelbarem Zusammenhang mit der Kernmembran (Abb. 2). Es durchzieht, oft mehrfach verzweigt, das generative Plasma und legt sich streckenweise so eng an die Zellwand, daß man eine Verbindung mit dieser vermutet. Eine Stelle, die den Übergang von endoplasmatischen Strukturen in die Wandung sicher zeigt, konnte jedoch nicht gefunden werden. Auf den Abbildungen erscheint das endoplasmatische Retikulum in dünnen Strängen, die dort, wo sie schräg durchschnitten sind, unklare Begrenzungslinien erkennen lassen. Serienschnitte beweisen, daß diese Stränge Platten darstellen, die eine Tiefe, also in der Ebene senkrecht zur Bildebene, bis zu mindestens $0,6-0,7 \mu$ erreichen. Diese Größenangabe, die nur als Abschätzung anzusehen ist, ergab sich bei der Auswertung der Beziehung zwischen Interferenzfarbe und Dicke eines Schnittes. Als maßgebende Tabellen für die Relation zwischen Interferenzfarbe und Schnittdicke dienten die Aufstellungen von Bachmans und Sitte $^{17}$ sowie die von Peachey ${ }^{18}$. Verzweigungen des endoplasmatischen Retikulums können tief in die Fortsätze der generativen Zelle hineinreichen und sich dort blasenartig verbreitern (Abb. 4). Häufig legen sich Elemente des endoplasmatischen Retikulums eng an Plastiden oder andere Zellorganellen an (Abb. 2, 7).

Plastiden: Verstreut im generativen Plasma liegen die etwa $1-2 \mu$ großen Plastiden (Abb. 2, 7). Sie zeigen den für undifferenzierte Plastiden typischen und schon mehrfach beschriebenen Bau (u. a.

17 L. Bachmaxi u. P. Sitte, Mikroskopie [Wien] 13, 289 [1958].

18 L. D. Peachey, J. biophysic. biochem. Cytol. 4, 233 [1958]. 


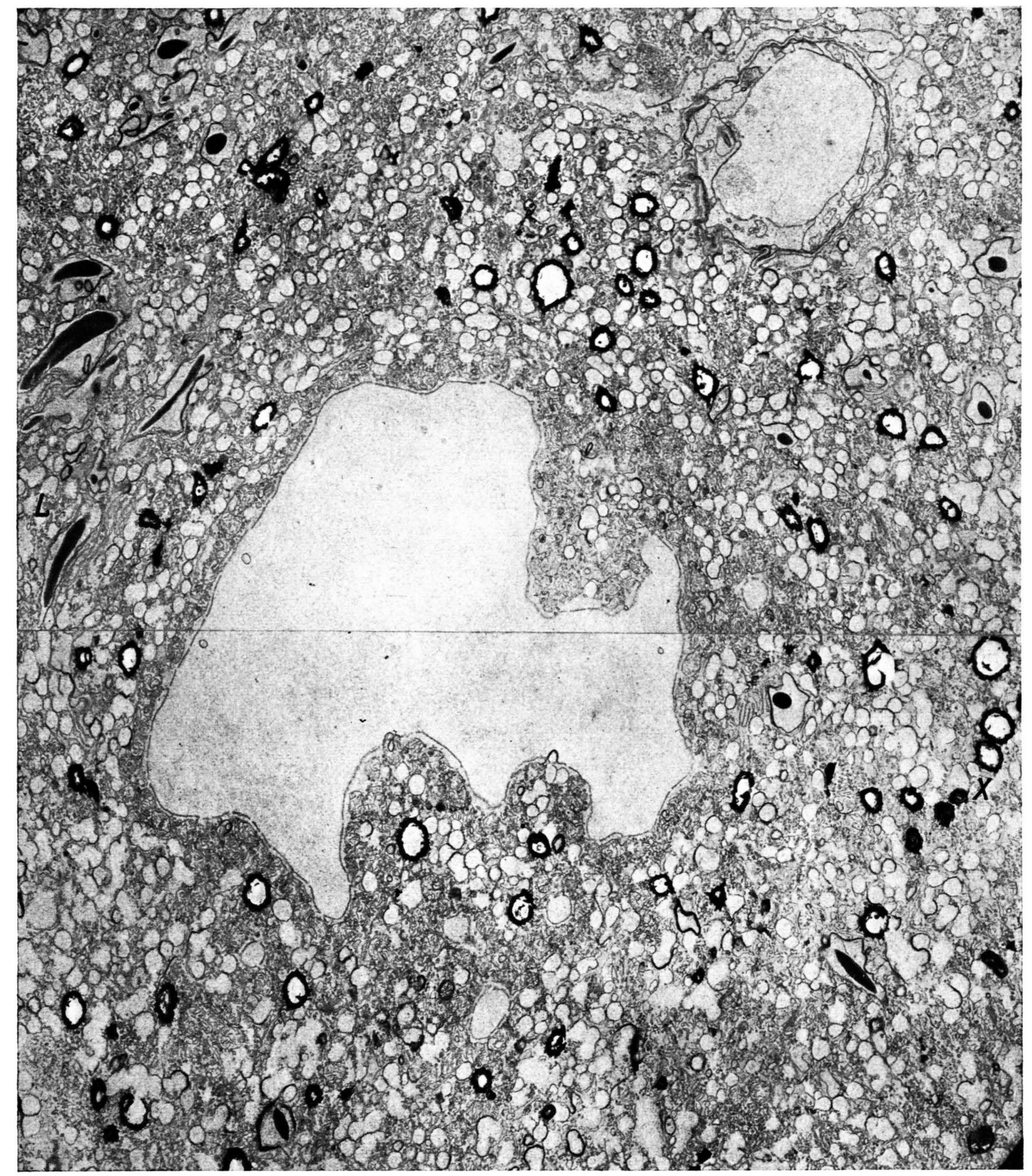

Abb. 1. Vegetativer Kern und generative Zelle im auskeimenden Pollenkorn. Im vegetativen Plasma Leukoplasten (L) mit Stärkekörnern sowie Lipoidtropfen (x). Vergr. ca. 5000 : l. (Das Bild wurde aus zwei Aufnahmen zusammengesetzt.) 


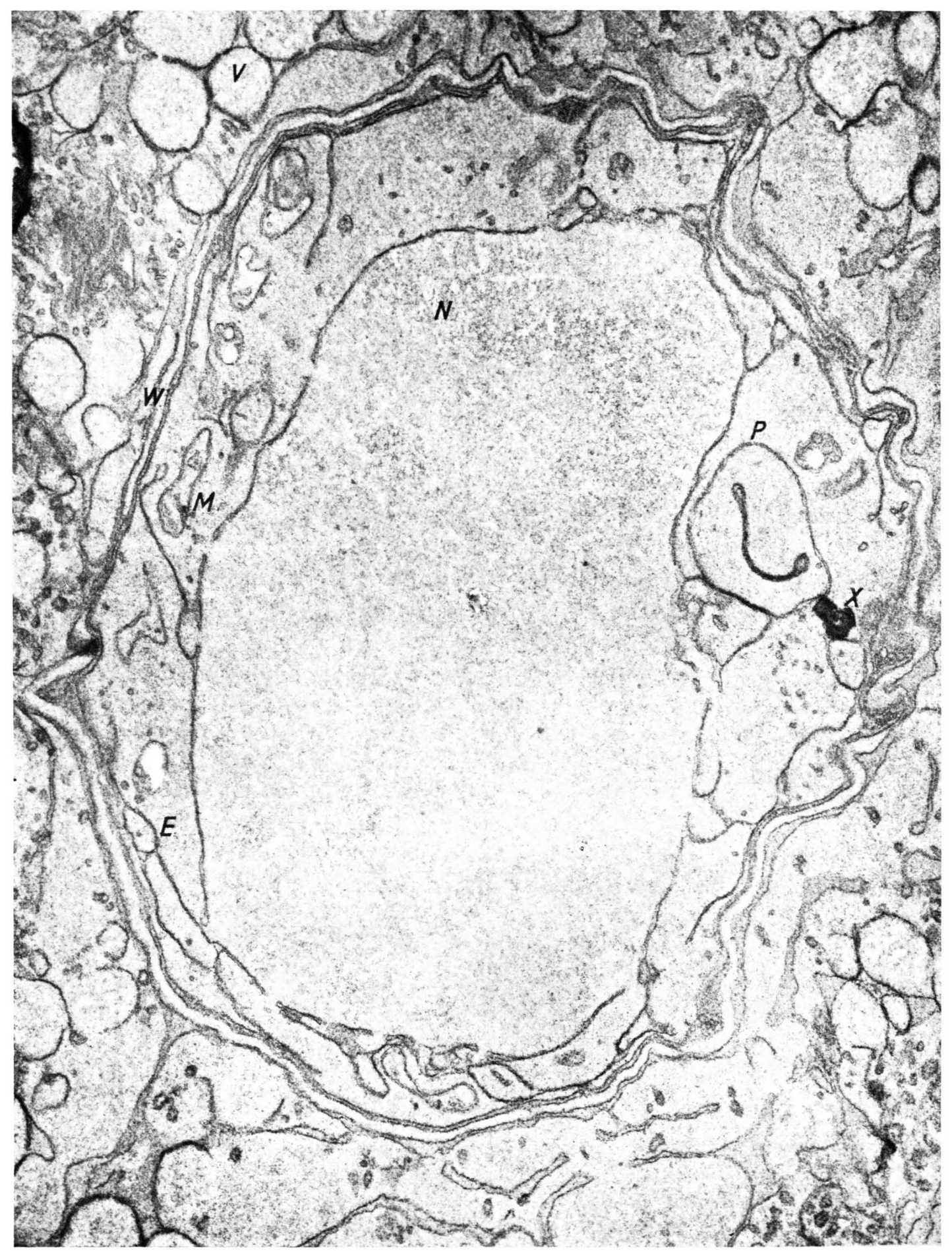

Abb. 2. Generative Zelle annähernd quer durchschnitten. E Endoplasmatisches Retikulum, M Mitochondrien, N Zellkern, P Plastiden, V Vakuolen im vegetativen Plasma, W Wandung der generativen Zelle, x Lipoidtropfen. Vergr. ca. 25000 : 1. 

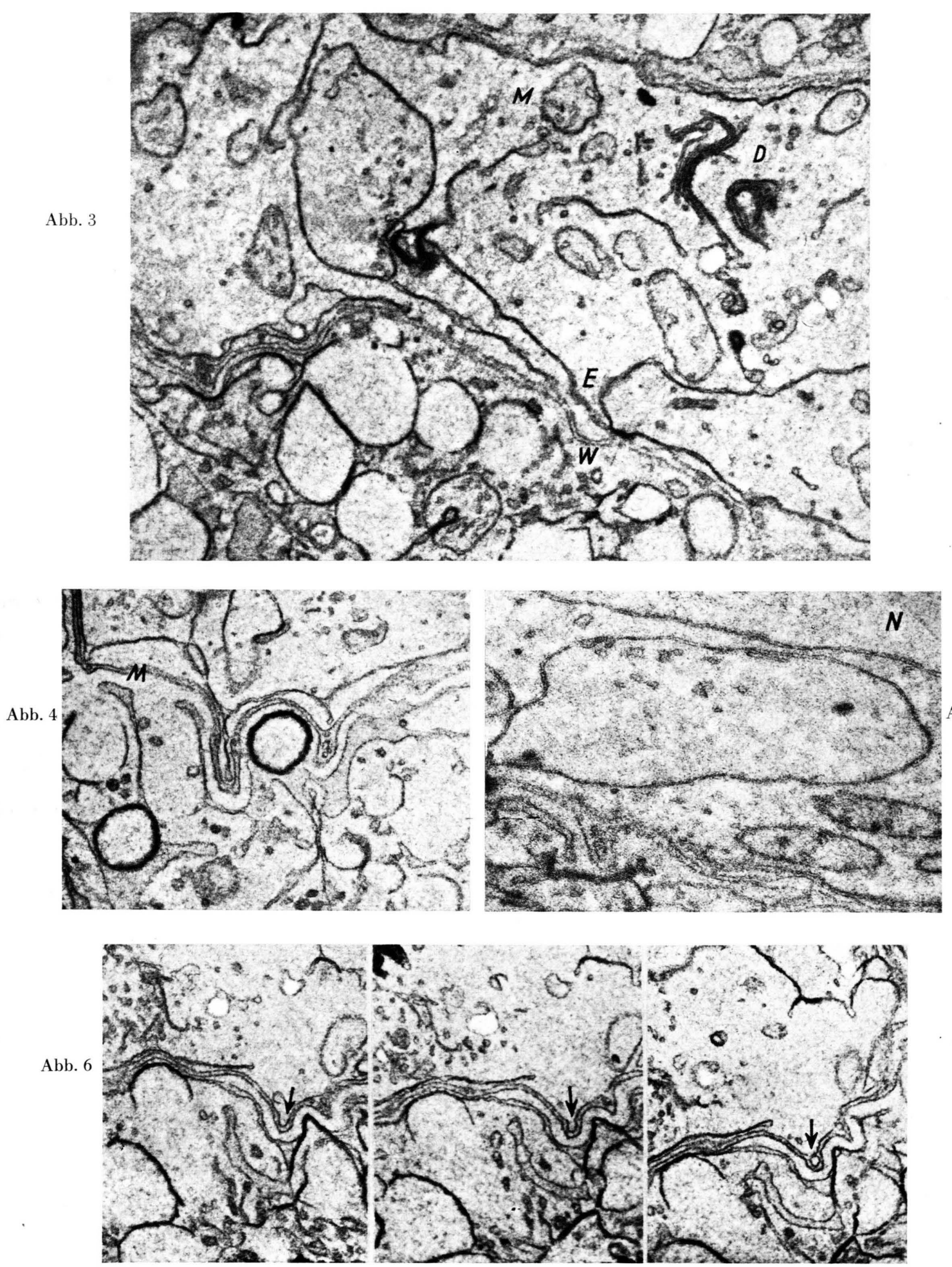

Abb. 3. Teil einer längsgeschnittenen generativen Zelle. D Diktyosomen. Vergr. ca. $25000: 1$.

Abb. 4. Fortsätze der generativen Zelle in das vegetative Plasma hinein. Vergr. ca. $25000: 1$.
Abb. 5. Plastide mit Doppellamelle. Vergr. ca. $39000: 1$. Abb. 6. Röhrchenartige Einstülpung des generativen Plasmas in die Wandung. Drei aufeinanderfolgende Schnitte. Der $\downarrow$ liegt im Innern der generativen Zelle. Vergr. ca. 25000 : 1. 


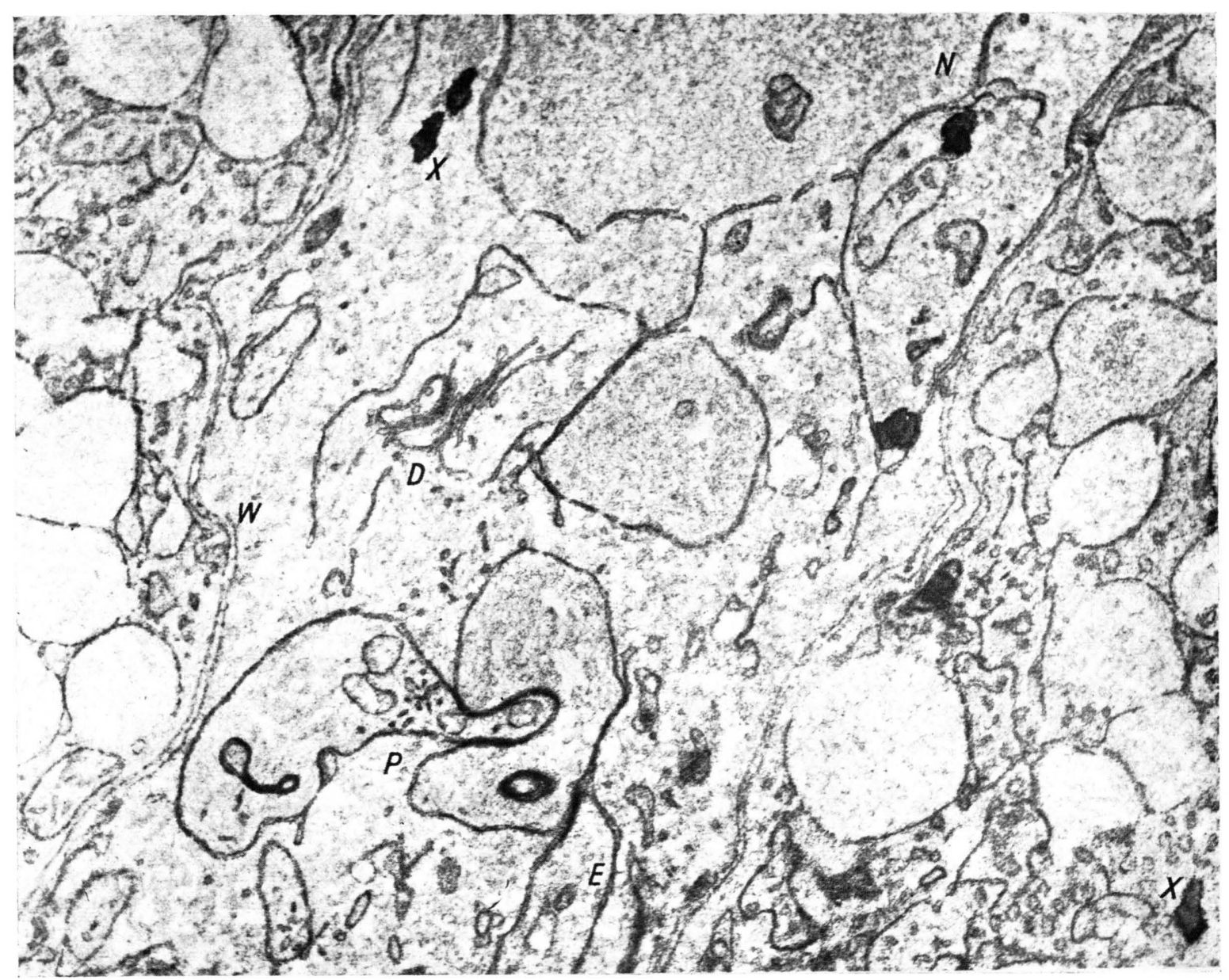

Abb. 7. Teil einer längsgeschnittenen generativen Zelle mit Plastiden-Zusammenlagerung. Vergr. ca. $29000: 1$.

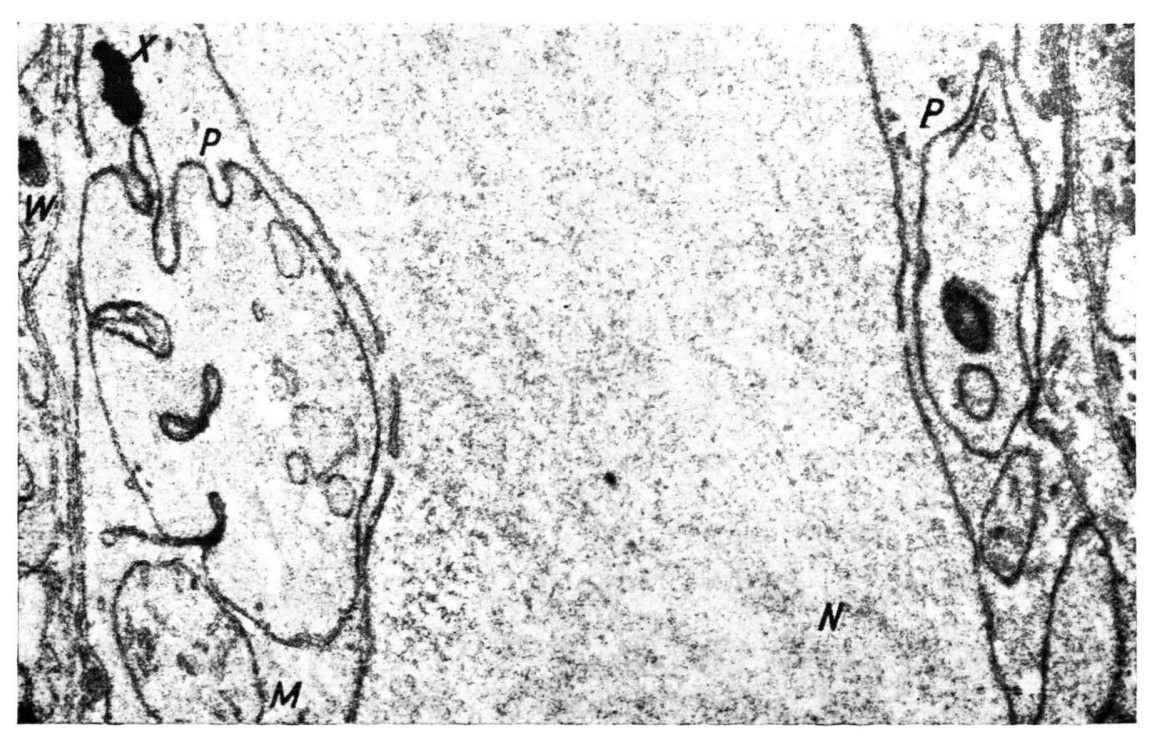

Abb. 8. Plastiden in generativer Zelle. Die rechts liegende Plastide enthält keine Stärke: der dunkle, ovale Fleck gehört zu einer tangential angeschnittenen Plasmaeinstülpung. Vergr. ca. $29000: 1$. 
Menke $^{19}$ ). Als Umgrenzungsmembran erscheinen zwei stark kontrastierte Linien, die eine hellere Zone einschließen. Im Innern der Plastiden erkennt man nur wenige Strukturen, vor allem kleine und gröBere blasenartige sowie kurze röhrenförmige Gebilde, die durch eine einfache Membran vom Stroma abgegrenzt sind. Anschnitte von Lamellen treten nur selten auf (Abb. 5). Häufig findet man doppelt konturierte, in sich geschlossene Streifen (Abb. 2, 7, 8) . Aus Serienschnitten folgt, daß ein solches Gebilde in den untersuchten Fällen die angeschnittene Umrandung einer Plasmaeinstülpung darstellt, die tief in das Plastideninnere hineinragen kann. Die Ausschnittsaufnahme einer längsgeschnittenen generativen Zelle (Abb. 7) zeigt eine Plastiden-Zusammenlagerung, bei der ein Fortsatz der einen Plastide weit in eine andere hineingreift. Ähnliche Figuren beschreibt Menke ${ }^{19}$ in Zellen des Vegetationskegels von Elodea canadensis. Diese Befunde sprechen für eine starke Verformbarkeit der undifferenzierten Plastiden, die sich ebenfalls in der oft amoeboiden Gestalt dieser Organellen äußert. - Manchmal liegen im Plastideninnern sehr helle Bereiche. Sie stellen zum Teil wohl Artefakte dar, die beim Schneidevorgang entstanden sind. Stärkekörner konnten niemals beobachtet werden. Prolamellarkörper, wie sie Hodge et al. ${ }^{20}$ beschrieben, wurden nicht gefunden, falls man nicht die selten vorkommenden Ansammlungen von einigen Tubuli oder Bläschen so bezeichnen will (Abb. 7). - Im reifen Pollenkorn von Oenothera hookeri stellte KaIEnBurg ${ }^{12}$ nach $\mathrm{AgNO}_{3}$ Imprägnierung sowie nach Régaud-Fixierung und anschließender Färbung mit Säurefuchsin viele $0,2-0,3 \mu$ große Gebilde innerhalb des generativen Plasmas fest, die sie als Plastiden ansieht. Diese Befunde lassen sich nach den elektronenmikroskopischen Bildern nicht bestätigen.

Mitochondrien: Die Mitochondrien im generativen Plasma sind verhältnismäßig leicht von den Plastiden zu unterscheiden, vor allem wenn man den Bau dieser beiden Organellen an Serienschnitten verfolgt. Die Mitochondrien erweisen sich mit einer durchschnittlichen Größe von etwa $0,5-1 \mu$ kleiner als die Plastiden. Sie zeigen den für Mitochondrien

19 W. MENKe, Z. Naturforschg. 15 b, 800 [1960].

20 A. J. Hodge, J. D. McLean and F. V. Mercer, J. biophysic. biochem. Cytol. 2, 597 [1956].

21 A. J. Daiton and M. D. Felix, J. biophysic. biochem. Cytol. 2 (suppl.), 79 [1956]. bekannten Bau (Abb. 3). Im Hinblick auf Größe der Organellen und Ausbildung ihrer Innenstruktur scheint bei Berücksichtigung aller untersuchten Präparate kein gesicherter Unterschied zwischen Mitochondrien des generativen und denen des vegetativen Plasmas zu bestehen.

Golgi-Strukturen und weitere Plasmabestandteile: Gelegentlich findet man Zusammenlagerungen von flachen, zum Teil gebogenen Platten, die senkrecht geschnitten wie kurze Schläuche aussehen. Nach Dalton und Felix ${ }^{21}$ sind sie als Diktyosomen zu bezeichnen. Es kommen außerdem im generativen Plasma Gebilde vor, die man nicht eindeutig als Golgi-Strukturen oder als Teile des endoplasmatischen Retikulums ansprechen kann.

Ferner treten sowohl in der generativen als auch in der vegetativen Zelle stark kontrastierte Einschlüsse (x) auf (Abb. 1, 2, 7, 8). Nach lichtmikroskopischen Untersuchungen an der vegetativen Zelle stellen sie wohl Lipoidtropfen dar, Menke ${ }^{19}$ beschrieb solche Gebilde in Sproßmeristemzellen von Elodea canadensis. $\mathrm{F}_{\mathrm{ALK}}{ }^{22}$ erwähnt sie in Wurzelmeristemzellen von Allium cepa und hält sie für Lipoidtropfen.

Das generative Plasma enthält noch einige Bestandteile, deren Zuordnung zu bekannten Organellen nicht geklärt ist. - Es besitzt keine Vakuolen der Größenordnung, wie sie in der vegetativen Zelle reichlich vertreten sind.

Nach den hier vorgelegten Ergebnissen zeigt die generative Zelle im Elektronenmikroskop einen Bau, der große Ähnlichkeit mit der Struktur bestimmter Zellen aus dem Sproß- und Wurzelmeristem aufweist, die bereits mehrfach, so von Sitte ${ }^{23}$, Buvat ${ }^{24}$, Lance ${ }^{25}$, Menke ${ }^{19}$, Whaley et al. ${ }^{16}$, FalK ${ }^{22}$ untersucht wurden.

\section{Zur Frage nach der Übertragung von Plasma- organellen bei der Befruchtung}

Im Zusammenhang mit einer Arbeit von RichterLandmann $^{26}$ sieht Bopp-Hassenkamp ${ }^{4}$ in ihrem Nachweis von Plastiden und Mitochondrien in der generativen Zelle eine wesentliche Stütze für die An-

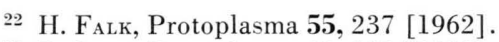

23 P. Sitte, Protoplasma 49, 447 [1958].

24 R. Buvat, Ann. Sci. natur., Bot. Biol. végétale 19, 121 [1958].

25 A. Lance, Ann. Sci. natur., Bot. Biol. végétale 19, 165 [1958]. 
sicht, daß Plasmaorganellen beim Befruchtungsvorgang in die Eizelle gelangen. Chardard ${ }^{4}$, der hingegen keine Plastiden und Mitochondrien in der generativen Zelle findet, weist auf die große Bedeutung seiner Ergebnisse für die außerkaryotische Vererbung hin, wenn seine Befunde sich als allgemeingültig erweisen sollten. - Auf die unserer Meinung nach nicht völlig überzeugenden lichtmikroskopischen Untersuchungen von Richter-LANDMANN ${ }^{26}$ an Impatiens glandulifera kann im einzelnen nicht eingegangen werden; es sei nur noch einmal auf die eingangs gegebenen Ausführungen über die Unterscheidbarkeit sehr kleiner Teilchen im Lichtmikroskop hingewiesen. - Aus dem Vorhandensein von Plasmapartikeln, wie z. B. Plastiden und Mitochondrien in der generativen Zelle, kann nicht geschlossen werden, daß sie schließlich auch in die Eizelle gelangen. Theoretisch bestehen nämlich für eine Übertragung von Organellen in die Eizelle folgende Möglichkeiten: 1. Nur der Kern aus der Spermazelle dringt ein. 2. Der gesamte Inhalt der Spermazelle gelangt in die Eizelle. 3. Beim Übertritt der Spermazelle bzw. des Spermakerns wird Plasma aus dem Pollenschlauch übertragen. Dabei ist noch zu bedenken, daß aus der Spermazelle oder dem Pollenschlauch in das Ei eingedrungenes Plasma dort oder im Embryo eliminiert werden kann. Diese Möglich-

26 W. Richter-Landmann, Planta 53, 162 [1959].

27 P. Michaelis, Planta 51, 722 [1958]. keit hängt nicht unmittelbar mit der cytologischen Frage nach dem Eindringen außerkaryotischer Teile in die Eizelle zusammen. Jedoch kommt ihr in genetischer Hinsicht Bedeutung zu. - Welche dieser drei Übertragungsarten gewinnt nun an Wahrscheinlichkeit, wenn man sie in Beziehung zu außerkaryotischen Vererbungserscheinungen setzt? Berücksichtigt man nur die Plastidengenetik, welche die meisten und sichersten Ergebnisse aufzuweisen hat, so ist bei Oenothera nach den Untersuchungen von Renner $^{7}$, Schwemmle und Mitarbb. ${ }^{8}, \mathrm{~S}_{\text {chötz }}{ }^{9}$, $\mathrm{S}_{\text {Tubbe }}{ }^{10}$ wohl mit dem Eindringen des Spermakerns zusammen mit Plasmateilen, zumindest Plastiden, zu rechnen. Dagegen besteht in der Gattung Epilobium nach Michaelis ${ }^{27}$ und bei Antirrhinum u. a. nach MALY ${ }^{28}$ durchaus die Möglichkeit, daß nur der Spermakern bei der Befruchtung übertragen wird; wenigstens läßt sich aus den genetischen Experimenten ein Übertritt von Plasmateilen nicht erkennen. - Aus den genetischen Befunden läßt sich also keine allgemeingültige Entscheidung zugunsten nur einer der aufgezählten Übertragungsweisen fällen.

Die vorliegende Arbeit wurde mit Unterstützung der Deutschen Forschungsgemeinschaft durchgeführt. Herrn Prof. Dr. W. Menke danke ich sehr für anregende Diskussionen und Hinweise.

28 R. Maly, Z. Vererbungsl. 89, 692 [1958]. 